\title{
Globe
}

Revue internationale d'études québécoises

\section{Introduction. Les enjeux du modèle des sociétés du savoir tels qu'ils sont vécus au Québec}

\section{Jean Bernatchez}

Volume 17, numéro 2, 2014

Les enjeux du modèle des sociétés du savoir tels qu'ils sont vécus au Québec

URI : https://id.erudit.org/iderudit/1036235ar

DOI : https://doi.org/10.7202/1036235ar

Aller au sommaire du numéro

Éditeur(s)

Globe, Revue internationale d'études québécoises

ISSN

1481-5869 (imprimé)

1923-8231 (numérique)

Découvrir la revue

Citer ce document

Bernatchez, J. (2014). Introduction. Les enjeux du modèle des sociétés du savoir tels qu'ils sont vécus au Québec. Globe, 17(2), 13-20.

https://doi.org/10.7202/1036235ar d'utilisation que vous pouvez consulter en ligne.

https://apropos.erudit.org/fr/usagers/politique-dutilisation/ 


\title{
INTRODUCTION. \\ LES ENJEUX DU MODĖLE \\ DES SOCIÉTÉS DU SAVOIR TELS QU'ILS SONT VÉCUS AU QUÉBEC
}

\author{
JEAN BERNATCHEZ \\ Université du Québec à Rimouski

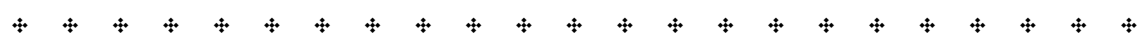

L'Organisation des Nations unies pour l'éducation, la science et la culture (UNESCO), dans son rapport Vers les sociétés du savoir publié en $2005^{1}$, propose un idéal inspirant, celui des sociétés du savoir. Une société qui aspire à devenir une "société du savoir" met en place des institutions et des politiques favorisant l'accès du plus grand nombre à la connaissance et à la pensée critique, ainsi que l'utilisation dans les pratiques sociales de savoirs scientifiques pertinents aux finalités de paix et de développement, selon des modalités associées au partage et à la collaboration.

Une société du savoir valorise la pluralité et la complémentarité des connaissances (savoirs scientifiques, techniques, traditionnels, pratiques, expérientiels, créatifs, etc.) et le libre accès aux connaissances savantes. Elle lutte contre les fractures sociales qui privent des citoyens de l'accès au savoir le plus élémentaire (la littératie). En 2012, l'Alliance mondiale pour la recherche au service de la communauté rédigeait une déclaration en ce sens, affirmant «que le droit à l'apprentissage, le droit à la connaissance, le droit à la production de connaissances, et le droit d'accéder à la connaissance constituent des droits inaliénables pour tous ${ }^{2} »$.

$$
\div \div
$$

1. UNESCO, Vers les sociétés du savoir, Paris, UNESCO, 2005.

2. Recherche partenariale du Canada (2015), "Alliance mondiale pour la recherche au service de la Communauté », http://communityresearchcanada.ca/?action=declar_fr (19 octobre 2015). 
Ce numéro de la revue Globe vise à mettre au jour le rôle que joue et que peut jouer la science dans la réalisation de cet idéal, au Québec plus particulièrement. À première vue, le rôle de l'institution scientifique (organisations, acteurs, pratiques, discours, ressources, connaissances, etc.) dans une société du savoir est crucial. Nourrie par la théorie, l'expérimentation ou la collecte de données, l'institution scientifique crée et diffuse les connaissances qui seront ensuite transmises à toute la société par le système d'éducation et les médias. Elle peut être un lieu fertile d'innovations qui transforment les conditions d'existence. Ce pouvoir lui impose la responsabilité de respecter, protéger et diffuser tous les savoirs imaginés et accumulés par l'Humanité, de viser l'universel de manière inclusive. Cet idéal doit cependant composer avec des contraintes.

Dans le premier type de contraintes, cet idéal s'oppose au modèle de l'économie du savoir proposé entre autres par l'Organisation de coopération et de développement économiques (OCDE) en 1996 dans son rapport L'économie fondée sur le savoir'. Ce modèle influence la plupart des politiques scientifiques nationales mises en œuvre au cours des deux dernières décennies. Il considère la recherche scientifique et l'innovation qu'elle génère comme un outil de développement de la compétitivité économique. Quand l'État considère le savoir comme un capital ou une marchandise et qu'il privilégie les sciences appliquées et les partenariats avec le secteur privé à but lucratif, que devient l'idéal des sociétés du savoir? Ce dilemme est clair dans le cas des universités québécoises : sont-elles des marchés compétitifs dont les clients sont les étudiants? Sont-elles un service public qui a pour mission de former des étudiants parce que la société a besoin de citoyens instruits et conscients de leur responsabilité sociale (médecins, enseignants, journalistes, ingénieurs, écrivains, biologistes, juristes, informaticiens, etc.) ?

Le deuxième type de contraintes est d'un autre ordre, même si on peut considérer qu'il fait partie des conséquences du mouvement vers le développement d'une économie du savoir. Il s'agit de la crise morale que traverse actuellement la pratique scientifique partout dans le monde, comme le montrent le nombre croissant de rétractations d'articles frauduleux même dans les plus grandes revues, le manque d'intérêt ou de confiance des décideurs dans les avis scientifiques, la fragilisation de l'autorité des organismes régulateurs (en sciences de la santé, notamment), les difficultés des sciences sociales et humaines à maintenir leur financement public, les conflits d'inté-

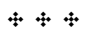

3. OCDE, L'économie fondée sur le savoir, Paris, OCDE, 1996. 
rêts individuels et institutionnels dans la recherche et l'innovation, le corporatisme et la domination d'un modèle unique de carrière scientifique, ainsi que la nouvelle "souffrance au travail» des chercheurs à qui on impose une productivité accrue. Comment, dans un tel contexte, encourager les chercheurs à s'engager dans l'avènement des sociétés du savoir? Et même, plus simplement, comment les encourager à s'en soucier?

Le troisième type de contraintes relève du fossé traditionnel entre la science et la société. D'un côté, la formation des chercheurs et la professionnalisation de leur "métier » les conduisent historiquement à privilégier comme public leurs pairs au détriment de la société en général (et, par exemple, à publier en anglais plutôt que dans la langue de leurs concitoyens). D'un autre côté, les citoyens hors de l'université (ou même les étudiants hors de leur discipline) se sentent incompétents relativement au savoir scientifique et osent rarement s'engager activement dans la véritable découverte d'un univers scientifique.

Le mouvement actuel qui se dessine dans de nombreux pays en faveur d'une science ouverte et le développement de la "troisième mission" des universités peuvent-ils constituer une voie fertile pour prendre contact avec l'idéal des sociétés du savoir? La science ouverte valorise l'accès libre et gratuit à toutes les publications scientifiques, le partage des connaissances et la collaboration entre chercheurs plutôt que la compétition et la marchandisation des publications et des innovations. La "troisième mission" des universités les conduit à encourager et à appuyer les relations concrètes entre leurs professeurs, leurs étudiants et la société civile afin que les savoirs des uns et des autres s'enrichissent mutuellement. Quels sont les choix des gouvernements, des législateurs, des universités et des chercheurs en matière d'" ouverture " de la science? Pour faire ce bilan, plusieurs chercheurs ont répondu à notre appel à contributions.

\section{PRÉSENTATION DES CONTRIBUTIONS}

Les deux premières contributions concernent des thèmes du rapport mondial de l'UNESCO sur les sociétés du savoir et la manière dont les universités au Québec se les sont appropriés.

Jean Bernatchez, de l'Université du Québec à Rimouski, présente d'abord un état des lieux au Québec de trois thèmes du rapport mondial de l'UNESCO sur les sociétés du savoir, dix ans après sa parution : la gouvernance de la science, l'organisation de la recherche scientifique et l'évolution de l'enseignement supérieur. Il s'inspire de l'approche cognitive d'analyse des politiques pour exposer les valeurs et les normes qui les orientent. En ce qui 
concerne la gouvernance de la science, l'UNESCO propose le modèle du public avec décisions partagées entre les lieux de la science, l'État, les entreprises et la société civile. L'auteur observe que l'action des gouvernements québécois et canadien va à l'encontre de ces principes, mais qu'il existe dans la société civile un tournant délibératif qui s'en inspire. Sur le plan de l'organisation de la recherche, le rapport rend compte de deux finalités comme vecteurs des sociétés du savoir : la paix et le développement, couplés à deux modalités, le partage et la collaboration. Au Québec, la recherche scientifique tend à être de plus en plus orientée, parfois dans un sens qui sert le bien commun, auquel cas ces initiatives relèvent surtout de l'engagement individuel de certains chercheurs. Enfin, l'évolution de l'enseignement supérieur montre que deux normes coexistent à travers le temps : celle d'association renvoie à une logique organisationnelle (le comment), tandis que la finalité éducative renvoie à une logique institutionnelle (le pourquoi). Il n'existe pas d'âge d'or de l'université au Québec, mais le mouvement actuel inspiré par le modèle de l'économie du savoir promu par l'OCDE implique une concentration des ressources dans les "grandes universités".

Les deux contributions qui suivent traitent d'expériences québécoises liées à la volonté de développer une société du savoir inclusive et caractérisée par l'idée de l'égalité des intelligences.

William-J. Beauchemin, Daniel Blémur, Nadia Duguay et Maxime Goulet-Langlois, praticiens-chercheurs associés à Exeko, un organisme d'innovation sociale de Montréal qui œuvre à l'inclusion des personnes, mettent de l'avant une approche qui reconnaît le potentiel de chaque individu, présume l'égalité des intelligences et repose sur le principe d'un savoir émancipateur. Leur démarche est fondée sur la médiation intellectuelle comme facteur de transformation sociale. Dans leur article, les quatre auteurs dressent le portrait de l'actuelle politique épistémologique basée sur la présupposition de l'inégalité des intelligences, une politique inspirée du modèle de l'économie du savoir promu par l'OCDE. Différents mécanismes sont à l'œuvre dans cette perspective, notamment le racisme de l'intelligence (un concept développé par Pierre Bourdieu), la subordination épistémologique (à cause de l'asservissement intellectuel, tel que le définit Jean-Pierre Darré), la société du mépris (thématisée par Jacques Rancière) et l'intériorisation de l'inégalité (avec le concept d'invisibilité sociale développé par Guillaume Le Blanc). Les auteurs militent pour l'émergence d'une nouvelle politique épistémologique fondée, cette fois-ci, sur l'égalité des intelligences et sur l'ouverture à tous de la position de sujet capable de science. La médiation intellectuelle contribue à cette entreprise : il s'agit d'une pratique philo- 
sophique, pédagogique et scientifique qui propose la création de situations égalitaires de réflexion collective et de partage des connaissances. Les auteurs rendent compte de leur expérience à ce chapitre, orientée par une pratique réflexive, critique et créative, comme lorsqu'ils exposent le cas d'un programme d'employabilité de jeunes autochtones. Ils croient que le caractère émancipateur d'une science ne se résume pas à ses résultats, mais s'étend à sa production. Une science ouverte doit être inclusive : il faut donc libérer la science du préjugé de l'inégalité des intelligences.

Cette volonté de développer une société du savoir inclusive et l'idée de l'égale capacité des personnes à comprendre une situation sont aussi les vecteurs de l'article de Marianne de Laat et Sophie Boyer du Mouvement ATD Quart Monde, associées à Catherine Hudon, Émilie Goulet et Christine Loignon de l'Université de Sherbrooke. Le Mouvement ATD Quart Monde lutte contre la pauvreté depuis 1957, année de sa création en France par Joseph Wresinski. En 1980, Wresinski invite les chercheurs universitaires qui s'intéressent au phénomène de la pauvreté à faire place à la connaissance qu'ont les pauvres de leur propre condition, affirmant ainsi que les personnes en situation d'exclusion et de pauvreté savent mieux que quiconque ce que doit être une société juste. Cette démarche de croisement des savoirs et des pratiques entre personnes en situation de pauvreté, universitaires et praticiens se traduit dans la recherche participative ÉQUIsanTÉ menée au sein de deux unités de médecine de famille de l'Université de Sherbrooke. Les auteures rendent d'abord compte de deux programmes pilotes franco-belges qui ont expérimenté les conditions de croisement des savoirs et des pratiques: Quart Monde Université (1996-1998) et Quart Monde Partenaire (1999-2001). Une Charte du croisement des savoirs et des pratiques a été rédigée à l'occasion de ces deux projets. Cette charte, couplée à une démarche d'application des connaissances, a jeté les bases de la recherche ÉQUIsanTÉ qui visait à améliorer la qualité et l'équité des soins offerts aux personnes en situation de pauvreté. La démarche de croisement des savoirs et des pratiques a été aussi importante que le savoir produit. Un espace collaboratif de construction des connaissances s'est développé à cette occasion.

Les deux autres contributions analysent des phénomènes propres au monde universitaire québécois.

Marie-Claude Bernard, de l'Université Laval, propose le récit de la mise en place d'une activité favorisant l'insertion universitaire des étudiants d'origine étrangère au Québec. Avec une volonté de circulation des savoirs rendue opératoire grâce à la mobilité internationale, l'Université Laval met 
en œuvre différentes stratégies d'internationalisation de ses activités. L'accueil d'étudiants d'autres pays pose le défi de leur intégration. L'article présente l'expérience de la création du cours L'insertion universitaire en milieu francophone nord-américain, conçu par l'auteure afin de relever ce défi. Une recherche documentaire et des rencontres avec des acteurs universitaires mettent d'abord en évidence certains aspects de la question : la maîtrise de la langue, la formation antérieure, la motivation et la persévérance scolaires, l'anxiété par rapport aux études, les méthodes de travail intellectuel, le rapport au temps et le décalage dans les compétences liées au métier d'étudiant. Trois pistes de réflexion sont ensuite amenées : les besoins des étudiants étrangers, les attentes produites par le système universitaire québécois et les thématiques qui doivent être abordées dans un cours à leur intention. Ce cours prévoit trois volets: les interventions pédagogiques à l'Université Laval, le processus d'apprentissage et la réflexion sur l'appartenance, l'identité et l'engagement dans la vie étudiante. L'expérience montre que ce qui est en jeu, ce ne sont pas seulement les compétences d'ordre cognitif, mais aussi la capacité des étudiants d'origine étrangère de s'adapter aux "allant de soi " et aux «non-dits» de la culture universitaire québécoise. Si l'université veut contribuer à l'édification d'une société du savoir inclusive, elle doit généraliser ce type d'initiatives et ne pas les réserver aux étudiants de premier cycle d'origine étrangère.

Jules Racine St-Jacques, de l'Université du Québec à Montréal, et Martin Maltais, de l'Université du Québec à Rimouski, s'intéressent pour leur part au financement public des universités au Québec, depuis la période d'après-guerre jusqu'à celle de la Révolution tranquille (1950-1968). En l'absence d'une véritable politique des universités, la dimension de leur financement permet de caractériser les grands vecteurs de leur évolution. Les années où Maurice Duplessis de l'Union nationale est le premier ministre du Québec sont marquées par un faible degré de normalisation des pratiques de financement des universités et un manque de planification du développement universitaire. Dans ce contexte, les universités du Québec ont comme mission implicite de régénérer la nation canadienne-française et catholique par la formation d'une élite professionnelle investie d'un devoir civilisateur et moral. Le financement des institutions comme l'université se présente ainsi, et souvent, comme une faveur accordée par le régime. Cela dit, une transition s'opère à compter de 1960, avec le gouvernement libéral du premier ministre Jean Lesage. Les universités se retrouvent alors au cœur d'un projet ambitieux de société démocratique. Certes, dans cette perspective, l'enseignement supérieur contribue au progrès social et économique par la forma- 
tion d'une main-d'œuvre hautement qualifiée, mais il offre aussi les clés d'une compréhension de l'univers social, économique culturel et naturel. Au cours de la période comprise entre 1950 et 1968, le mode d'allocation des ressources aux universités s'est donc transformé: il est passé d'un mode discrétionnaire à un mode normalisé, avec comme objectif explicite d'inscrire l'université au cœur du développement social et économique du Québec. Toutefois, malgré ces efforts de systématisation, les traits d'une culture discrétionnaire persistent à la fin des années 1960.

Nancy Emond, de l'Université Laval, donne ensuite dans une note de recherche un aperçu de la pratique de la science ouverte dans les universités francophones du Québec. Elle définit le concept de science ouverte comme une nouvelle forme de pratique de la science basée sur les principes constitutifs des sociétés du savoir, soit une production commune de connaissances par des chercheurs de disciplines variées et par des acteurs non scientifiques, une production dont les résultats sont accessibles gratuitement. Elle relève que, sur les 736 unités de recherche qu'elle a analysées dans huit universités, $52 \%$ sont formées de chercheurs provenant de plus d'une discipline et $40 \%$, de plus d'une université. En moyenne, $35 \%$ de ces unités pratiquent la science ouverte. Elle remarque des différences entre les universités et les domaines de recherche. Par exemple, la science ouverte est davantage pratiquée à l'Université du Québec à Rimouski (60\%) qu'à l'Université de Montréal (17\%); elle est aussi plus pratiquée en sciences humaines et sociales ( $44 \%$ ) qu'en sciences de la santé $(24 \%)$. En outre, les unités de recherche établies en région pratiquent davantage la science avec des acteurs de la société civile. Les unités de recherche multidisciplinaires et interuniversitaires effectuent davantage leurs travaux avec des acteurs non universitaires. Le modèle de l'économie du savoir fait peser sur l'autre modèle, celui des sociétés du savoir, une pression pour une privatisation plus grande des résultats de la recherche et une productivité accrue. Cette résistance au partage du savoir ne provient-elle pas des producteurs de connaissances eux-mêmes, puisque, dans ce monde orienté vers la compétitivité économique, détenir "en exclusivité » un savoir procure un pouvoir non négligeable?

L'étude libre éclaire en outre l'influence du Québec sur une dimension importante de la politique étrangère du Canada. Justin Massie, de l'Université du Québec à Montréal, et David G. Haglund, de la Queen's University, proposent une analyse de l'influence du Québec sur les interventions militaires canadiennes. Ils retiennent deux cas qui ont largement dominé les relations canado-américaines des récentes années : la guerre en 
Afghanistan et l'invasion anglo-américaine de l'Irak. Il ressort entre autres de leur analyse que les Québécois expriment des préférences distinctes de celles des autres Canadiens en s'opposant de manière forte à une participation militaire canadienne à ces conflits. 